\title{
Existence of positive radial solution for Neumann problem on the Heisenberg group
}

\section{F. Safari ${ }^{1}$ and A. Razani ${ }^{1 *}$ (D)}

\section{"Correspondence:}

razani@sci.ikiu.ac.ir

${ }^{1}$ Department of Pure Mathematics, Faculty of Science, Imam Khomeini

International University, Qazvin, Iran

\section{Abstract}

The existence of at least one positive radial solution of the Neumann problem

$$
-\Delta_{\mathbb{H}^{n}} u+R(\xi) u=a\left(|\xi|_{\mathbb{H}^{n}}\right)|u|^{p-2} u-b\left(|\xi|_{\mathbb{H}^{n}}\right)|u|^{q-2} u,
$$

is proved on the Heisenberg group $\mathbb{H}^{n}$, via the variational principle, where $a\left(|\xi|_{\mathbb{H}^{n}}\right)$, $b\left(|\xi|_{\mathbb{H}^{n}}\right)$ are nonnegative radial functions and $R(\xi)$ satisfies suitable conditions.

MSC: 35R03; 35J20; 46E35

Keywords: Heisenberg group; Variational principle; Supercritical Neumann problem; Mountain Pass Geometry theorem; Palais-Smale compactness condition

\section{Introduction}

Semilinear elliptic equations were the first nonlinear generalization of linear elliptic partial differential equations. They are of fundamental importance for the study of engineering, geometry, life sciences, economics, physics and mechanics; see [1, 23-27]. Some examples, like the theory of Bose-Einstein condensates, obey

$$
-\Delta u=f(x, u) .
$$

The variational approach to these equations has experienced spectacular success in recent years, reaching a high level of complexity and refinement, with a multitude of applications (see $[10,14-16,22])$.

In [3] and [12] some problems which depend on continuous component of time like coherent states in quantum optics are probed. These problems are studied in a space which have a component of time and are known as Heisenberg group. Important topics where the Heisenberg group reveals itself as an essential factor are quantum mechanics, ergodic theory, representation theory of nilpotent Lie group, foundation of abelian harmonic analysis, and the theory of partial differential equations. We are now interested in the last one.

(c) The Author(s) 2020. This article is licensed under a Creative Commons Attribution 4.0 International License, which permits use, sharing, adaptation, distribution and reproduction in any medium or format, as long as you give appropriate credit to the original author(s) and the source, provide a link to the Creative Commons licence, and indicate if changes were made. The images or other third party material in this article are included in the article's Creative Commons licence, unless indicated otherwise in a credit line to the material. If material is not included in the article's Creative Commons licence and your intended use is not permitted by statutory regulation or exceeds the permitted use, you will need to obtain permission directly from the copyright holder. To view a copy of this licence, visit http://creativecommons.org/licenses/by/4.0/ 
In this paper, the existence of at least one radial solution of a generalized well-known supercritical Neumann problem

$$
\begin{cases}-\Delta_{\mathbb{H}^{n}} u+R(\xi) u=a\left(|\xi|_{\mathbb{H}^{n}}\right)|u|^{p-2} u-b\left(|\xi|_{\mathbb{H}^{n}}\right)|u|^{q-2} u & \xi \in \Omega, \\ u>0 & \xi \in \Omega, \\ \frac{\partial u}{\partial n}=0 & \xi \in \partial \Omega,\end{cases}
$$

is proved where $\Omega$ is the Korányi ball in the Heisenberg group. Recently, the existence of a nontrivial weak solution of following singular boundary value problem on the Heisenberg group was proved (see [29]):

$$
\begin{cases}-\Delta_{\mathbb{H}^{n}} u=\mu \frac{g(\xi) u}{\left(|z|^{4}+t^{2}\right)^{\frac{1}{2}}}+\lambda f(\xi, t) & \xi \in \Omega, \\ u=0 & \xi \in \partial \Omega .\end{cases}
$$

In [18] problem (1.1) is studied where $R(\xi)=1$ on the unit ball in $\mathbb{R}^{n}, n \geq 2$, see $[2,17,21$, 22] for some applications.

Here we recall some definitions and results on the Heisenberg group (see $[4,7,30])$. Let us recall some features of the Heisenberg group. The Heisenberg group $\mathbb{H}^{n}=\left(\mathbb{R}^{2 n+1}, 0\right)$ is the space $\mathbb{R}^{2 n+1}$ with the noncommutative law of product

$$
(x, y, t) \circ\left(x^{\prime}, y^{\prime}, t^{\prime}\right)=\left(x+x^{\prime}, y+y^{\prime}, t+t^{\prime}+2\left(\left\langle y, x^{\prime}\right\rangle-\left\langle x, y^{\prime}\right\rangle\right)\right),
$$

where $x_{1}, x_{2}, y_{1}, y_{2} \in \mathbb{R}^{n}, t_{1}, t_{2} \in \mathbb{R}$ and $\langle$,$\rangle denotes the standard inner product in \mathbb{R}^{n}$. This operation endows $\mathbb{H}^{n}$ with the structure of a Lie group. The Lie algebra of $\mathbb{H}^{n}$ is generated by the left-invariant vector fields

$$
T=\frac{\partial}{\partial t}, \quad X_{i}=\frac{\partial}{\partial x_{i}}+2 y_{i} \frac{\partial}{\partial t}, \quad Y_{i}=\frac{\partial}{\partial y_{i}}-2 x_{i} \frac{\partial}{\partial t}, \quad i=1,2,3, \ldots, n .
$$

These generators satisfy the noncommutative formula

$$
\left[X_{i}, Y_{j}\right]=-4 \delta_{i j} T, \quad\left[X_{i}, X_{j}\right]=\left[Y_{i}, Y_{j}\right]=\left[X_{i}, T\right]=\left[Y_{i}, T\right]=0 .
$$

Let $z=(x, y) \in \mathbb{R}^{2 n}$ and $\xi=(z, t) \in \mathbb{H}^{n}$. The parabolic dilation

$$
\delta_{\lambda} \xi=\left(\lambda x, \lambda y, \lambda^{2} t\right)
$$

satisfies

$$
\delta_{\lambda}\left(\xi_{0} \circ \xi\right)=\delta_{\lambda} \xi \circ \delta_{\lambda} \xi_{0},
$$

and

$$
|\xi|_{\mathbb{H}^{n}}=\left(|z|^{4}+t^{2}\right)^{\frac{1}{4}}=\left(\left(x^{2}+y^{2}\right)^{2}+t^{2}\right)^{\frac{1}{4}},
$$


is a norm with respect to the parabolic dilation which is known as Korányi gauge norm $N(z, t)$. The Heisenberg distance between two points $(z, t)$ and $\left(z^{\prime}, t^{\prime}\right)$ is given by

$$
\rho\left(z, t ; z^{\prime}, t^{\prime}\right)=\left|\left(z^{\prime}, t^{\prime}\right)^{-1} \circ(z, t)\right|_{\mathbb{H}^{n}}
$$

Clearly, the vector fields $X_{i}, Y_{i}, i=1,2, \ldots, n$ are homogeneous of degree 1 under the norm $|\cdot|_{\mathbb{H}^{n}}$ and $T$ is homogeneous of degree 2 . The Korányi ball of center $\xi_{0}$ and radius $r$ is defined by

$$
B_{\mathbb{H}^{n}}\left(\xi_{0}, r\right)=\left\{\xi:\left|\xi^{-1} \circ \xi_{0}\right|_{\mathbb{H}^{n}} \leq r\right\}
$$

and it satisfies

$$
\left|B_{\mathbb{H}^{n}}\left(\xi_{0}, r\right)\right|=\left|B_{\mathbb{H}^{n}}(0, r)\right|=r^{2 n+2}\left|B_{\mathbb{H}^{n}}(0,1)\right| .
$$

The Heisenberg gradient and Kohn-Laplacian (Heisenberg-Laplacian) operator on $\mathbb{H}^{n}$ are given by

$$
\nabla_{\mathbb{H}^{n}}=\left(X_{1}, X_{2}, \ldots, X_{n}, Y_{1}, Y_{2}, \ldots, Y_{n}\right)
$$

and

$$
\Delta_{\mathbb{H}^{n}}=\sum_{i=1}^{n} X_{i}^{2}+Y_{i}^{2}
$$

respectively. Let $\Omega \subset \mathbb{H}^{n}, n \geq 1$, be a smooth bounded open set. Its associated Sobolev space is defined as follows:

$$
H^{1}\left(\Omega, \mathbb{H}^{n}\right):=\left\{u \in L^{2}(\Omega): X_{i} u, Y_{i} u \in L^{2}(\Omega), i=1,2, \ldots, n\right\},
$$

and closure of $C_{0}^{\infty}(\Omega)$ in $H^{1}\left(\Omega, \mathbb{H}^{n}\right)$ under the norm

$$
\|u\|_{H^{1}\left(\Omega, \mathbb{H}^{n}\right)}=\left(\int_{\Omega}\left(\left|\nabla_{\mathbb{H}^{n}} u\right|^{2}+|u|^{2}\right) d \xi\right)^{\frac{1}{2}}
$$

where $u: \Omega \subset \mathbb{H}^{n} \rightarrow \mathbb{R}$, is denoted by $H_{0}^{1}\left(\Omega, \mathbb{H}^{n}\right)$. The following norm is a norm on $H_{0}^{1}\left(\Omega, \mathbb{H}^{n}\right)$ :

$$
\|u\|_{H_{0}^{1}\left(\Omega, \mathbb{H}^{n}\right)}=\left(\int_{\Omega}\left|\nabla_{\mathbb{H}^{n}} u\right|^{2} d \xi\right)^{\frac{1}{2}}
$$

which is equivalent to the standard one. The dual space of $H_{0}^{1}(\Omega, \mathbb{H})$ will be denoted by $H^{-1}(\Omega, \mathbb{H})$. Here we recall Hardy's inequality and some results on the Heisenberg group.

Lemma 1.1 ([19]) For $n \geq 1$ and for any $u \in H_{0}^{1}\left(\Omega, \mathbb{H}^{n}\right)$, we have

$$
\int_{\Omega} \frac{|u|^{2}}{\left(|z|^{4}+|t|^{2}\right)^{\frac{1}{2}}} d \xi \leq\left(\frac{n+1}{n^{2}}\right)^{2} \int_{\Omega} \mid \nabla_{\left.\mathbb{H}^{n} u\right|^{2}} d \xi
$$


For convenience, we write the above inequality as follows:

$$
\int_{\Omega} \frac{|u|^{2}}{\left(|z|^{4}+|t|^{2}\right)^{\frac{1}{2}}} d \xi \leq \frac{1}{C_{n}} \int_{\Omega}\left|\nabla_{\mathbb{H}^{n}} \mathcal{u}\right|^{2} d \xi
$$

where $C_{n}=\left(\frac{n^{2}}{n+1}\right)^{2}$.

Lemma $1.2([13,29])$ Let $\Omega \subset \mathbb{H}^{n}$ be a bounded open set. Then the following compact embedding is satisfied:

$$
H_{0}^{1}\left(\Omega, \mathbb{H}^{n}\right) \subset \subset L^{s}(\Omega) \quad \text { for } 1 \leq s<\bar{Q},
$$

where $\bar{Q}=\frac{2 Q}{Q-2}$ is critical exponent of $Q=2 n+2$, which is a homogeneous dimension of $\mathbb{H}^{n}$. We denote the Sobolev embedding constant of the above compact embedding by $C_{s}>0$; i.e.

$$
\|u\|_{L^{s}(\Omega)} \leq C_{s}\|u\|_{H_{0}^{1}\left(\Omega, \mathbb{H}^{n}\right)} \quad \text { for all } u \in H_{0}^{1}(\Omega, \mathbb{H}), 1 \leq s \leq \bar{Q}
$$

Consider two subspaces of the Heisenberg-Sobolev space $H_{0}^{1}(\Omega, \mathbb{H})$, as follows:

$$
D^{1,2}(\Omega):=\left\{u: \Omega \rightarrow \mathbb{R}: u,\left|\nabla_{\mathbb{H}^{n}} u\right| \in L^{2}(\Omega)\right\}
$$

with respect to the norm

$$
\|u\|_{D^{1,2}(\Omega)}=\left(\int_{\Omega}\left(\left|\nabla_{\mathbb{H}^{n}} u\right|^{2}+|u|^{2}\right) d \xi\right)^{\frac{1}{2}},
$$

and $D_{0}^{1,2}(\Omega)$ be the closure of $C_{0}^{\infty}(\Omega)$ in $\left\{u \in D^{1,2}(\Omega): 0 \leq u, u\right.$ is increasing $\}$. According to Poincarés inequality in Heisenberg-Sobolev space (see [8]), $D_{0}^{1,2}(\Omega)$ is equipped with the norm

$$
\|u\|_{D_{0}^{1,2}(\Omega)}=\left(\int_{\Omega}\left(\left|\nabla_{\mathbb{H}^{n}} u\right|^{2}\right) d \xi\right)^{\frac{1}{2}}
$$

which is equivalent to the standard one. Additionally, define

$$
D^{2,2}(\Omega):=\left\{u: \Omega \rightarrow \mathbb{R}: u,\left|\nabla_{H} u\right|,\left|\Delta_{H} u\right| \in L^{2}(\Omega)\right\}
$$

which is equipped with the norm

$$
\|u\|_{D^{2,2}(\Omega)}=\left(\int_{\Omega}\left(\left|\Delta_{\mathbb{H}^{n}} u\right|^{2}+\left|\nabla_{\mathbb{H}^{n}} u\right|^{2}+|u|^{2}\right) d \xi\right)^{\frac{1}{2}}
$$

The following compact embedding is needed for the main result.

Theorem 1.1 ([9]) Let $\Omega \subset \mathbb{H}^{n}$ be a bounded open set. Then the following embeddings are compact:

(I) If $Q=4$, then $D^{2,2}(\Omega) \cap D_{0}^{1,2}(\Omega) \hookrightarrow L^{s}(\Omega), 1 \leq s<\infty$. 
(II) If $Q>4$, then $D^{2,2}(\Omega) \cap D_{0}^{1,2}(\Omega) \hookrightarrow L^{s}(\Omega), 1 \leq s<Q^{*}$, where $Q^{*}=\frac{2 Q}{Q-4}$ and as above $Q=2 n+2$.

Now we recall results we need later. Let $V$ be a real Banach space and $V^{*}$ be its topological dual and pairing between $V$ and $V^{*}$ denoted by $\langle$,$\rangle . A function \psi: V \rightarrow \mathbb{R}$ is called weakly lower semi-continuous (w.l.s.c.) if

$$
\psi(u) \leq \lim _{n \rightarrow \infty} \inf \psi\left(u_{n}\right)
$$

for each $u \in V$ and any sequence $\left\{u_{n}\right\}$ converging to $u$ in the weak topology $\sigma\left(V, V^{*}\right)$. Let $\psi: V \rightarrow \mathbb{R} \cup\{+\infty\}$ be a proper convex function. The subdifferential of $\psi$ at $u$ is the set

$$
\partial \psi(u)=\left\{u^{*} \in V^{*}: \psi(v) \geq \psi(u)+\left\langle u^{*}, v-u\right\rangle \text { for all } v \in V\right\}
$$

The multivalued mapping $\partial \psi: V \rightarrow 2^{V^{*}}$ (where $2^{V^{*}}$ is the set of all subsets of $V^{*}$ ) by $\psi$ is Gâteaux differentiable at $u$, denoted by $D \psi(u)$, if and only if $\partial \psi(u)$ is a singleton. In this case, $\partial \psi(u)=\{D \psi(u)\}$.

Lemma 1.3 ([1]) Let $X$ be a reflexive Banach space and $I: X \rightarrow \mathbb{R}$ be a continuous convex functional. Then I is w.l.s.c..

Theorem 1.2 (Weierstrass, [1]) Let $X$ be a reflexive Banach space and $I: X \rightarrow \mathbb{R}$ be w.l.s.c. and coercive. Then I has a global minimum point.

Definition 1.1 Let $V$ be a real Banach space, $\varphi \in C^{1}(V, \mathbb{R})$ and $\psi: V \rightarrow(-\infty,+\infty]$ be a proper (i.e. Dom $\psi \neq \emptyset$ ), convex and lower semi-continuous function, and $K$ be a convex and weakly closed subset of $V$. Define the function $\psi_{K}: V \rightarrow(-\infty,+\infty]$ by

$$
\psi_{K}(u):= \begin{cases}\psi(u) & u \in K \\ +\infty & u \notin K .\end{cases}
$$

Suppose the functional I: $V \rightarrow(-\infty,+\infty]$ is defined by

$$
I(w):=\psi_{K}(w)-\varphi(w)
$$

$u \in \operatorname{Dom}\left(\psi_{K}\right)$ is called a critical point of $I$, if $D \varphi(u) \in \partial \psi_{K}(u)$ or equivalently,

$$
\psi_{K}(v)-\psi_{K}(u) \geq\langle D \varphi(u), v-u\rangle, \quad \text { for all } u \in V \text {. }
$$

Notice that a global minimum point of $I$ is a critical point of $I$.

Definition 1.2 ([11]) We say that I satisfies the Palais-Smale compactness condition (PS) if every sequence $\left\{u_{n}\right\}$ is such that

- $I\left[u_{n}\right] \rightarrow c \in \mathbb{R}$,

- $\left\langle D \varphi\left(u_{n}\right), u_{n}-v\right\rangle+\psi(v)-\psi\left(u_{n}\right) \geq-\varepsilon_{n}\left\|v-u_{n}\right\|$, for all $v \in V$, where $\varepsilon_{n} \rightarrow 0$, then $\left\{u_{n}\right\}$ possesses a convergent subsequence. 
The following Mountain Pass Geometry (MPG) theorem is proved in [28].

Theorem 1.3 Suppose that $I: V \rightarrow(-\infty,+\infty]$ is of the form (1.3) and satisfies the PS condition and the following conditions:

(i) $I(0)=0$,

(ii) there exists $e \in V$ such that $I(e) \leq 0$,

(iii) there exists positive constant $\rho$ such that $I(u)>0$, if $\|u\|=\rho$.

Then I has a critical value $c \leq \rho$ which is characterized by

$$
c=\inf _{g \in \Gamma} \sup _{t \in[0,1]} I[g(t)]
$$

where $\Gamma=\{g \in C([0,1], V): g(0)=0, g(1)=e\}$.

Subsequently, we need the following theorem.

Theorem 1.4 (G. Polya, [20]) Assume that $u_{n}:[a, b] \rightarrow \mathbb{R}, n \geq 1$ is a monotone increasing sequence of (not necessarily continuous) functions which converge point-wise to a continuous function $u:[a, b] \rightarrow \mathbb{R}$, then the convergence is uniform.

The first eigenvalue plays a very relevant role in nonlinear elliptic problems.

Remark 1.1 (see [1]) According to the variational characterization of the first eigenvalue when $\lambda_{1}=\lambda_{1}\left(-\Delta_{\mathbb{H}^{n}}+R\right)>0$ (this happens certainly if $R(\xi) \geq 0$ a.e. in $\Omega$ ) or when $R$ satisfies one of the conditions of Theorem 1.5, it is easy to see that the quantity

$$
(u \mid v)=\int \nabla_{\mathbb{H}^{n}} u \nabla_{\mathbb{H}^{n}} v d \xi+\int R(\xi) u v d \xi
$$

defines a scalar product on $H_{0}^{1}\left(\Omega, \mathbb{H}^{n}\right)$ that induces a norm

$$
\|u\|_{*}=\left(\left.\int\left|\nabla_{\left.\mathbb{H}^{n} u\right|^{2}}+R(\xi)\right| u\right|^{2} d \xi\right)^{\frac{1}{2}}
$$

equivalent to the standard one and similarly on $D_{0}^{1,2}(\Omega)$.

Now we can state the main result of this paper.

Theorem 1.5 Let $\Omega$ be a smooth, bounded open set in the Heisenberg group $\mathbb{H}^{n}, 2 \leq q<$ $p<Q^{*}$ and $a, b \in L^{\infty}(0,1)$ be increasing positive non-constant radial functions. The problem (1.1) admits at least one radially increasing solution if at least one of the following conditions is met:

(i) $R(\xi)=\alpha$ where $\alpha>0$ is a real constant.

(ii) $R(\xi) \in L^{\infty}(\Omega)$ and $\lambda_{1}\left(-\Delta_{\mathbb{H}^{n}}+R(\xi)\right) \geq 0$, where $\lambda_{1}$ is the first eigenvalue.

(iii) $R(\xi) \leq c\left(1+|\omega|_{\mathbb{H}^{n}}\right)^{-2}$, where $c \geq 0$ is a small enough constant.

\section{Positive radial solution}

Here, we recall the following variational principle established in [5]. 
Definition 2.1 The triple $(\psi, \varphi, K)$ satisfies the point-wise invariance condition at a point $u \in V$ if there exists a convex Gâteaux differentiable function $G: V \rightarrow \mathbb{R}$ and a point $v \in K$ such that

$$
D \psi(v)+\mathrm{DG}(v)=D \varphi(u)+\mathrm{DG}(u) .
$$

Theorem 2.1 Let $V$ be a reflexive Banach space and $K$ be a convex and weakly closed convex subset of $V$. Let $\psi: V \rightarrow \mathbb{R} \cup\{+\infty\}$ be a convex, lower semi-continuous function which is Gâteaux differentiable on $K$, and let $\varphi \in C^{1}(V, \mathbb{R})$. Assume that the following two assertions hold:

(i) The functional $I: V \rightarrow \mathbb{R} \cup\{+\infty\}$ defined by $I(w)=\psi_{K}(w)-\varphi(w)$ has a critical point $u \in V$ as in Definition 2.1,

(ii) the triple $(\psi, \varphi, K)$ satisfies the point-wise invariance condition at the point $u$.

Then $u \in K$ is a solution of the equation

$$
D \psi(u)=D \varphi(u)
$$

To use Theorem 2.1, consider the bounded open set $\Omega \subset \mathbb{H}^{n}$ and the Banach space $V=D_{0, r}^{1,2}(\Omega) \cap D^{2,2}(\Omega) \cap L_{a}^{p}(\Omega) \cap L_{b}^{q}(\Omega)$, where

$$
L_{a}^{p}(\Omega)=\left\{u: \int_{\Omega} a\left(|\xi|_{\mathbb{H}^{n}}\right)|u|^{p} d \xi<\infty\right\}
$$

and

$$
L_{b}^{q}(\Omega)=\left\{u: \int_{\Omega} b\left(|\xi|_{\mathbb{H}^{n}}\right)|u|^{q} d \xi<\infty\right\}
$$

and also

$$
D_{0, r}^{1,2}(\Omega)=\left\{u \in D_{0}^{1,2}(\Omega): 0 \leq u, u \text { is a radial function }\right\}
$$

$D^{2,2}(\Omega)$ is defined as above and $u: \Omega \rightarrow \mathbb{R}$ is called radial function if $u(x, y, t)=\phi(\varrho)$ where $\varrho=|(x, y, t)|_{\mathbb{H}^{n}} . V$ is equipped with the norm

$$
\begin{aligned}
\|u\|_{V}:= & \|u\|_{*}+\|u\|_{L_{a}^{p}}+\|u\|_{L_{b}^{q}} \\
= & \left(\int_{\Omega}\left|\nabla_{\mathbb{H}^{n}} u\right|^{2}+R(\xi)|u|^{2} d \xi\right)^{\frac{1}{2}}+\left(\int_{\Omega} a\left(|\xi|_{\mathbb{H}^{n}}\right)|u|^{p} d \xi\right)^{\frac{1}{p}} \\
& +\left(\int_{\Omega} b\left(|\xi|_{\mathbb{H}^{n}}\right)|u|^{q} d \xi\right)^{\frac{1}{q}} .
\end{aligned}
$$

We consider the Euler-Lagrange functional corresponding to problem (1.1); i.e.

$$
E(u)=\frac{1}{2} \int_{\Omega}\left|\nabla_{\mathbb{H}^{n}} u\right|^{2}+R(\xi)|u|^{2} d \xi+\frac{1}{q} \int_{\Omega} b\left(|\xi|_{\mathbb{H}^{n}}\right)|u|^{q} d \xi-\frac{1}{p} \int_{\Omega} a\left(|\xi| \mathbb{H}^{n}\right)|u|^{p} d \xi,
$$

over the convex and weakly closed set

$$
K=\left\{u \in V: u \geq 0, u \text { is increasing with respect to the radius } \varrho=|\omega|_{\mathbb{H}^{n}}\right\} \text {. }
$$


To adapt Theorem 2.1 to our problem, we define $\psi, \varphi: V \rightarrow \mathbb{R}$ by

$$
\psi(u)=\frac{1}{2} \int_{\Omega}\left|\nabla_{\mathbb{H}^{n}} u\right|^{2}+R(\xi)|u|^{2} d \xi+\frac{1}{q} \int_{\Omega} b\left(|\xi|_{\mathbb{H}^{n}}\right)|u|^{q} d \xi
$$

and

$$
\varphi(u)=\frac{1}{p} \int_{\Omega} a\left(|\xi|_{\mathbb{H}^{n}}\right)|u|^{p} d \xi .
$$

Finally, let us introduce the functional $I: V \rightarrow(-\infty,+\infty]$ as follows:

$$
I(u)=\psi_{K}(u)-\varphi(u),
$$

where $\psi_{K}$ is defined as (1.2). We should be aware that $I$ is indeed the Euler-Lagrange functional corresponding to our problem restricted to $K$. Theorem 2.1 implies the following corollary.

Corollary 2.1 Let $V=D_{0, r}^{1,2}(\Omega) \cap D^{2,2}(\Omega) \cap L_{a}^{p}(\Omega) \cap L_{b}^{q}(\Omega)$, and $K$ be the convex closed subset of $V$ given in (2.1). Assume that the functional

$$
I(w):=\psi_{K}(w)-\frac{1}{p} \int_{\Omega} a\left(|\xi|_{\mathbb{H}^{n}}\right)|w|^{p} d \xi
$$

has a critical point $u \in V$ and there exists $v \in K$ satisfying the linear equation

$$
\begin{cases}-\Delta_{H} v+R(\xi) v+b\left(|\xi|_{\mathbb{H}^{n}}\right)|v|^{q-2} v=a\left(|\xi|_{\mathbb{H}^{n}}\right)|u|^{p-2} u & \xi \in \Omega, \\ \frac{\partial v}{\partial n}=0 & \xi \in \partial \Omega\end{cases}
$$

in the weak sense. Then $u \in K$ is a solution of the equation

$$
\begin{cases}-\Delta_{H} u+R(\xi) u+b\left(|\xi|_{\mathbb{H}^{n}}\right)|u|^{q-2} u=a\left(|\xi|_{\mathbb{H}^{n}}\right)|u|^{p-2} u & \xi \in \Omega, \\ \frac{\partial u}{\partial n}=0 & \xi \in \partial \Omega .\end{cases}
$$

Notice that satisfying Eq. (2.3) shows that the triple $\left(\psi_{K}, \varphi, K\right)$ satisfies the point-wise invariance condition at $u$, where $G=0$. We need some lemmas and theorems before proving our main result.

Lemma 2.1 Assume $R(\xi)$ is given in Theorem 1.5. If $h \in L^{2}(\Omega), f: \mathbb{R} \rightarrow \mathbb{R}$ is continuous and there exist $\alpha, \beta>0$ such that

$$
|f(t)| \leq \alpha+\beta|t|^{Q^{*}-1} \quad \text { and } \quad f(t) t \leq 0
$$

for all $t \in \mathbb{R}$. Then the problem

$$
\begin{cases}-\Delta_{\mathbb{H}^{n}} u+R(\xi) u=f(u)+h(\xi) & \xi \in \Omega \\ \frac{\partial u}{\partial n}=0 & \xi \in \partial \Omega\end{cases}
$$

admits at least one solution. 
Proof First notice that by integration, there exist $\alpha_{1}, \beta_{1}>0$ such that

$$
|F(t)| \leq \alpha_{1}+\beta_{1}|u|^{Q^{*}}
$$

and $F(t) \leq 0$ for all $t \in \mathbb{R}$, where $F(t)=\int_{0}^{t} f(s) d s$. Now consider the following energy functional corresponding to problem (2.4) on $D_{0}^{1,2}(\Omega) \cap D^{2,2}(\Omega)$ :

$$
J(u)=\frac{1}{2}\|u\|_{*}^{2}-\int_{\Omega} F(u) d x-\int_{\Omega} h u d x .
$$

Theorem 1.1 implies

$$
J(u) \geq \frac{1}{2}\|u\|_{*}^{2}-C\|u\|_{*} .
$$

So $J$ is coercive and weakly lower semi-continuous on $D_{0}^{1,2}(\Omega)$. So according to the Weierstrass theorem, $J$ has a global minimum point which means problem (2.4) admits at least one solution.

Lemma 2.2 For $2 \leq q<p<Q^{*}$, there exists $C>0$ such that

$$
\|u\|_{*} \leq\|u\|_{V} \leq C\|u\|_{*}
$$

for all $u \in K$.

Proof Theorem 1.1 implies

$$
\begin{aligned}
\|u\|_{*} & \leq\|u\|_{V}=\|u\|_{*}+\left(\int_{\Omega} a\left(|\xi|_{\mathbb{H}^{n}}\right)|u|^{p} d \xi\right)^{\frac{1}{p}}+\left(\int_{\Omega} b\left(|\xi|_{\mathbb{H}^{n}}\right)|u|^{q} d \xi\right)^{\frac{1}{q}} \\
& \leq\|u\|_{*}+C_{1}^{\prime}|a|_{L^{\infty}(\Omega)}\|u\|_{D_{0}^{1,2}(\Omega)}+C_{2}^{\prime}|b|_{L^{\infty}(\Omega)}\|u\|_{D_{0}^{1,2}(\Omega)} \\
& \leq\left(1+C_{1}+C_{2}\right)\|u\|_{*} .
\end{aligned}
$$

The last estimate holds, since $\|\cdot\|_{*}$ and $\|\cdot\|_{D_{0}^{1,2}(\Omega)}$ are equivalent.

Lemma 2.3 Let $V=D_{0, r}^{1,2}(\Omega) \cap D^{2,2}(\Omega) \cap L_{a}^{p}(\Omega) \cap L_{b}^{q}(\Omega)$. Suppose the functional $I: V \rightarrow \mathbb{R}$ is defined by

$$
I(u):=\psi_{K}(u)-\varphi(u)
$$

where $\varphi$ and $\psi_{K}$ are given in Corollary 2.1. Then I has a nontrivial critical point.

Proof We utilize Theorem 1.3 to prove this lemma. First recall that

$$
D \varphi(u)=a\left(|\xi|_{\mathbb{H}^{n}}\right)|u|^{p-2} u,
$$

and therefore $\varphi$ is a $C^{1}$-function on the space $V$. Also notice that $\psi$ is a proper, convex and lower semi-continuous and $K$ is closed in $V$. We are going to prove this lemma in two steps: 
Step 1. We verify that $I$ satisfies in MPG conditions. It is clear that $I(0)=0$. Take $e \in K$. It follows that

$$
I(t e)=\frac{t^{2}}{2}\|u\|_{*}^{2}+\frac{t^{q}}{q} \int_{\Omega} b\left(|\xi|_{\mathbb{H}^{n}}\right)|e|^{q} d \xi-\frac{t^{p}}{p} \int_{\Omega} a\left(|\xi|_{\mathbb{H}^{n}}\right)|e|^{p} d \xi
$$

Now since $p>q \geq 2$, for $t$ sufficiently large $I(t e)$ is negative. We now prove condition (iii) of the MPG theorem. Take $u \in \operatorname{Dom}(\psi)$ with $\|u\|_{V}=\rho>0$. Notice that from Lemma 2.2 for $u \in K$ we have

$$
\|u\|_{V} \leq\left(1+C_{1}+C_{2}\right)\|u\|_{*} .
$$

Also

$$
\begin{aligned}
& \varphi(u)=\frac{1}{p} \int_{\Omega} a\left(|\xi|_{\mathbb{H}^{n}}\right)|u|^{p} d \xi \leq C_{3}\|u\|_{V}^{p}=C_{3} \rho^{p}, \\
& I(u) \geq \frac{\rho^{2}}{2\left(1+C_{1}+C_{2}\right)^{2}}-C_{3} \rho^{p}>0,
\end{aligned}
$$

provided $\rho>0$ is small enough as $2<p$ and $C_{1}, C_{2}, C_{3}$ are positive constants. If $u \notin$ $\operatorname{Dom}(\psi)$. Then clearly $I(u)>0$. Therefore, MPG holds for the functional $I$.

Step 2. We verify the PS compactness condition. Suppose that $\left\{u_{n}\right\}$ is a sequence in $K$ such that $I\left(u_{n}\right) \rightarrow c \in \mathbb{R}, \epsilon_{n} \rightarrow 0$ and

$$
\left\langle D \varphi\left(u_{n}\right), u_{n}-v\right\rangle+\psi_{K}(v)-\psi_{K}\left(u_{n}\right) \geq-\epsilon_{n}\left\|v-u_{n}\right\|_{V}, \quad \text { for all } v \in V \text {. }
$$

We show that $\left\{u_{n}\right\}$ has a convergent subsequence in $V$. First notice that $u_{n} \in \operatorname{Dom}(\psi)$, then

$$
I\left(u_{n}\right)=\psi_{K}\left(u_{n}\right)-\varphi\left(u_{n}\right) \rightarrow c, \quad \text { as } n \rightarrow \infty .
$$

Thus, for large values of $n$ we have

$$
\psi_{K}\left(u_{n}\right)-\varphi\left(u_{n}\right) \leq 1+c .
$$

Now consider the function $g(r)=r^{q}-p(r-1)-1$ on the interval $(1,+\infty)$ and set $r^{*}=\left(\frac{p}{q}\right)^{\frac{1}{q-1}}$. It is easy to see that for every $r \in\left(1, r^{*}\right)$ we have $g(r)<0$. We choose a number $r$ for which we have $r>1$ and $r^{q}-1<p(r-1)$. In (2.7) set $v=r u_{n}$. Then

$$
(1-r)\left\langle D \varphi\left(u_{n}\right), u_{n}\right\rangle+\left(r^{q}-1\right) \psi_{K}\left(u_{n}\right) \geq-\epsilon_{n}(r-1)\left\|u_{n}\right\|_{V} .
$$

Furthermore,

$$
\left\langle D \varphi\left(u_{n}\right), u_{n}\right\rangle=\int_{\Omega} a\left(|\xi|_{\mathbb{H}^{n}}\right) u_{n}(\xi)^{p} d \xi=p \varphi\left(u_{n}\right) .
$$

Since $r^{q}-1<p(r-1)$, we can take $\beta>0$ such that $\frac{1}{p(r-1)}<\beta<\frac{1}{r^{q}-1}$. Multiplying (2.9) by $\beta$ and adding it to (2.8) we obtain

$$
[1-\beta p(1-r)] \varphi\left(u_{n}\right)+\left[1-\beta\left(r^{q}-1\right)\right] \psi_{K}\left(u_{n}\right) \leq 1+c+\beta C\left\|u_{n}\right\|_{*} .
$$


So for a suitable constant $C^{\prime}>0$

$$
\left\|u_{n}\right\|_{*}^{2} \leq \psi_{K}\left(u_{n}\right) \leq C^{\prime}\left(1+\left\|u_{n}\right\|_{*}\right)
$$

Therefore, $\left\{u_{n}\right\}$ is a bounded sequence in Hilbert space $D_{0, r}^{1,2}(\Omega) \cap D^{2,2}(\Omega)$. Theorem 1.1 and the standard results in Sobolev space show there exists $\bar{u} \in D_{0, r}^{1,2}(\Omega) \cap D^{2,2}(\Omega)$ such that, up to subsequences,

- $u_{n} \rightarrow \bar{u}$ in $D_{0, r}^{1,2}(\Omega) \cap D^{2,2}(\Omega)$;

- $u_{n} \rightarrow \bar{u}$ in $L^{s}(\Omega), 1 \leq s<Q^{*}$;

- $u_{n}(\xi) \rightarrow \bar{u}(\xi)$ a.e in $\Omega$;

- there exist $w_{s} \in L^{s}(\Omega)$ such that $\left|u_{n}(\xi)\right| \leq w_{s}(\xi)$ a.e. in $\Omega$ and for all $n \in \mathbb{N}$ where $1 \leq s<Q^{*}$.

Now in (2.7) replace $v$ with $\bar{u}$ :

$$
-\int_{\Omega} a\left(|\xi|_{\mathbb{H}^{n}}\right)\left|u_{n}\right|^{p-1}\left(\bar{u}-u_{n}\right) d \xi+\psi_{K}(\bar{u})-\psi_{K}\left(u_{n}\right) \geq-\epsilon_{n}\left\|\bar{u}-u_{n}\right\|_{V}
$$

On the one hand, we have

$$
\left.\left|\int_{\Omega} a\left(|\xi|_{\mathbb{H}^{n}}\right)\right| u_{n}\right|^{p-1}\left(\bar{u}-u_{n}\right) d \xi\left|\leq \int_{\Omega} a\left(|\xi|_{\mathbb{H}^{n}}\right) w_{p-1}^{p-1}(\xi)\right| \bar{u}-u_{n} \mid d \xi
$$

On the other hand, $\left\{u_{n}\right\} \subset K$ so $\left\{u_{n}\right\}$ is an increasing sequence so $\left\{\left|\bar{u}-u_{n}\right|\right\}$ is an increasing sequence, too. Now according to Theorem 1.4 the right hand side of the latter inequality goes to zero. Therefore passing into limits in (2.11), one gets

$$
\lim _{n \rightarrow \infty} \sup \psi_{K}\left(u_{n}\right) \leq \psi_{K}(\bar{u})
$$

But the norm is weakly lower semi-continuous and $u_{n} \rightarrow \bar{u}$ in $L_{b}^{q}(\Omega)$. So $\psi_{K}\left(u_{n}\right) \leq$ $\lim _{n \rightarrow \infty} \inf \psi_{K}\left(u_{n}\right)$. Then

$$
\lim _{n \rightarrow \infty} \psi_{K}\left(u_{n}\right)=\psi_{K}(\bar{u})
$$

Then $\left\|u_{n}\right\|_{V} \rightarrow\|\bar{u}\|_{V}$, and besides $u_{n}(\xi) \rightarrow \bar{u}(\xi)$ a.e. thus $u_{n} \rightarrow \bar{u}$ strongly in $V$ as desired. Notice that every $u_{n}$ is radial, so $u$ is radial; moreover, $u \in K$.

Lemma 2.4 Suppose $u \in K$. There exists $v \in K$ such that

$$
\begin{cases}-\Delta_{\mathbb{H}^{n}} v+R(\xi) v=a\left(|\xi|_{\mathbb{H}^{n}}\right) u^{p-1}-b\left(|\xi|_{\mathbb{H}^{n}}\right) v^{q-1} & \text { in } \Omega, \\ v=0 & \text { on } \partial \Omega,\end{cases}
$$

in the weak sense.

Proof Let $u \in K$ so $0 \leq u \in K \subset D^{2,2}$. According to Theorem 2.4, it is enough to show that $h(\xi)=a\left(|\xi|_{\mathbb{H}^{n}}\right) u(\xi)^{p-1}$ belongs to $L^{2}(\Omega)$ and $f(v)=-b\left(|\xi|_{\mathbb{H}^{n}}\right) v(\xi)^{q-1}$ satisfies the inequality

$$
|f(t)| \leq \alpha+\beta|t|^{Q^{*}-1} \text { and } f(t) t \leq 0
$$


for all $t \in \mathbb{R}$. Clearly since $q<Q^{*}$ both hold. On the other hand, as $u \in L^{Q^{*}}(\Omega)$ and $\Omega \subset \mathbb{H}^{n}$ one can see following estimate:

$$
\begin{aligned}
|u(\xi)| & =|\phi(\varrho)|=\left|\int_{0}^{\varrho} \phi^{\prime}(s) d s+\phi(0)\right| \\
& \leq \int_{0}^{\varrho}\left|\phi^{\prime}(s)\right| d s+|\phi(0)| \\
& \leq\left(\int_{0}^{\varrho}\left|\phi^{\prime}(s)\right|^{2} s^{2 n} d s\right)^{\frac{1}{2}}\left(\int_{0}^{\varrho} \frac{d s}{s^{2 n}}\right)^{\frac{1}{2}}+|\phi(0)| \\
& \leq C\left(\omega_{2 n}^{-1} \int_{\Omega}\left|\phi^{\prime}(\xi)\right|^{2} d \xi\right)^{\frac{1}{2}} \varrho^{\frac{1-2 n}{2}}+|\phi(0)| \\
& \leq C^{\prime}\left(\int_{\Omega} \frac{\left|\nabla_{\mathbb{H}^{n}} u\right|^{2}}{|\xi|_{\mathbb{H}^{n}}^{2}} d \xi\right)^{\frac{1}{2}}\left(\int_{\Omega} \xi^{2} d \xi\right)^{\frac{1}{2}} \varrho^{\frac{1-2 n}{2}}+|\phi(0)| \\
& \leq C^{\prime \prime}|\xi|_{\mathbb{H}^{n}}^{2-n} \frac{1}{C_{n}} \int_{0}^{\varrho}\left|\Delta_{\mathbb{H}^{n}} u\right|^{2} d \xi+|\phi(0)| \\
& \leq C_{*}\|u\|_{D^{2,2}(\Omega)}|\xi|_{\mathbb{H}^{n}}^{2-n},
\end{aligned}
$$

where $\omega_{2 n}$ is the measure of unit ball in $\mathbb{R}^{2 n+1}=\mathbb{H}^{n}$, and $C, C^{\prime}, C^{\prime \prime}, C_{*}$ are positive constants, and the fundamental theorem of calculus and Hardy's inequality (1.1) are applied. Recent inequalities are satisfied since $u$ is a radial function so $\left|\nabla_{\mathbb{H}^{n}} u\right|=\frac{\varrho}{r}\left|\phi^{\prime}\right|$ where $\varrho=|\xi|_{\mathbb{H}^{n}}=$ $|(z, t)|_{\mathbb{H}^{n}}, r=|z|$ (see [6]). Because of the last inequality and since $p<Q^{*}$, it is easy to see that $h \in L^{2}(\Omega)$. This means the desired result has been obtained.

Now we can state the proof of Corollary 2.1, by using Theorem 2.1 and introducing an appropriate space.

Proof Let $V=D_{0, r}^{1,2}(\Omega) \cap D^{2,2}(\Omega) \cap L_{a}^{p}(\Omega) \cap L_{b}^{q}(\Omega)$. By introducing the norm

$$
\begin{aligned}
\|u\|_{V}:= & \|u\|_{*}+\|u\|_{L_{a}^{p}}+\|u\|_{L_{b}^{q}} \\
= & \left(\int_{\Omega}\left|\nabla_{\mathbb{H}^{n}} u\right|^{2}+R(\xi)|u|^{2} d \xi\right)^{\frac{1}{2}}+\left(\int_{\Omega} a\left(|\xi|_{\mathbb{H}^{n}}\right)|u|^{p} d \xi\right)^{\frac{1}{p}} \\
& +\left(\int_{\Omega} b\left(|\xi|_{\mathbb{H}^{n}}\right)|u|^{q} d \xi\right)^{\frac{1}{q}}
\end{aligned}
$$

on $V$, it is converted to a reflexive Banach space. Note that

$$
K=\left\{u \in V: \frac{\partial u}{\partial n}=0 \text { on } \partial \Omega \text { and } u \text { is a radially increasing function }\right\}
$$

is a convex closed subset and we define the functional $I=\psi_{K}-\varphi$ which is the EulerLagrange functional restricted to $K$. It follows from the MPG theorem and the PS compactness condition in Lemma 2.3 that the functional $I$ has a critical point $u$, also it is guaranteed that $I(u)>0$. Lemma 2.4 implies that there exists $v \in \operatorname{Dom}(\psi)$ satisfying the linear equation $D \psi_{K}(v)=D \varphi(u)$. Setting $C(\xi):=1-a\left(|\xi|_{\mathbb{H}^{n}}\right) u(\xi)^{p-1}$ we have $-\Delta_{\mathbb{H}^{n}} u+C(\xi) u=0$ in $\Omega$. We show $u>0$ in $\Omega$. Otherwise, by applying the strong maximum principle, we 
deduce $u$ is identically zero in $\Omega$. On the other hand $u$ is a radially increasing function. This a contradiction and so $u$ must be a nontrivial nonnegative solution of (1.1). So the conditions of Corollary 2.1 hold and the proof is completed.

Remark 2.1 Corollary 2.1 implies problem (1.1) has at least one nontrivial radially increasing solution.

Acknowledgements

We thank the anonymous referees for their valuable comments, which improved the paper.

Funding

Not available.

Availability of data and materials

Not applicable.

Competing interests

The authors have declared that no competing interests exist.

Authors' contributions

The authors contributed equally to this paper. All authors read and approved the final manuscript.

\section{Authors' information}

${ }^{1}$ Department of Pure Mathematics, Faculty of Science, Imam Khomeini International University, Qazvin, Iran. E-mails:

f.safari@edu.ikiu.ac.ir, razani@sci.ikiu.ac.ir.

\section{Publisher's Note}

Springer Nature remains neutral with regard to jurisdictional claims in published maps and institutional affiliations.

Received: 30 March 2020 Accepted: 29 April 2020 Published online: 12 May 2020

\section{References}

1. Badiale, M., Serra, E.: Semilinear Elliptic Equations for Beginners, Existence Results via the Variational Approach. Springer, Berlin (2011)

2. Bahrouni, A., Radulescu, V.: On a new fractional Sobolev space and applications to nonlocal variational problems with variable exponent. Discrete Contin. Dyn. Syst., Ser. S 11(3), 379-389 (2018)

3. Band, Y.B., Avishai, Y.: Quantum Mechanics with Applications to Nanotechnology and Information Science. Academic Press, San Diego (2012)

4. Birindelli, I., Ferrari, F., Valdinoci, E.: Semilinear PDEs in the Heisenberg group: the role of the right invariant vector fields. Nonlinear Anal. 72, 987-997 (2010)

5. Cowan, C., Moameni, A.: A new variational principle, convexity and supercritical Neumann problems. Trans. Am. Math. Soc. 371, 5993-6023 (2019)

6. D'Ambrosio, L.: Some Hardy inequalities on the Heisenberg group. J. Differ. Equ. 40, 552-564 (2004)

7. Domokos, A.: On the regularity of $p$-harmonic functions in the Heisenberg group. Doctoral Dissertation, University of Pittsburgh (2004)

8. Dong, X.-Y., Lu, G.-Z., Sun, L.-J.: Global Poincaré inequalities on the Heisenberg group and applications. Acta Math. Sin. Engl. Ser. 23, 735-744 (2007)

9. Dwivedi, G., Tyagi, J.: Stability of positive solutions to biharmonic equations with logistic-type nonlinearities on Heisenberg group (2019) arXiv:1606.06413v4

10. Ekeland, I., Temam, R: Convex Analysis and Variational Problems. SIAM, Philadelphia (1999)

11. Evans, L.: Partial Differential Equations, Sobolev Spaces. Academic Press, New York (1998)

12. Everett, S.: A (Mostly) Coherent Talk on Heisenberg Group (Coherent States and Their Applications in Physics). University of California, Santa Cruz (2017)

13. Garofalo, N., Lanconelli, E.: Existence and nonexistence results for semilinear equations on the Heisenberg group. Indiana Univ. Math. J. 41(1), 71-98 (1992)

14. Makvand Chaharlang, M., Razani, A.: A fourth order singular elliptic problem involving $p$-biharmonic operator. Taiwan J. Math. 23(3), 589-599 (2019)

15. Makvand Chaharlang, M., Razani, A.: Existence of infinitely many solutions for a class of nonlocal problems with Dirichlet boundary condition. Commun. Korean Math. Soc. 34(1), 155-167 (2019)

16. Makvand Chaharlang, M., Razani, A.: Two weak solutions for some Kirchhoff-type problem with Neumann boundary condition. Georgian Math. J. (2020) 10 pages. https://doi.org/10.1515/gmj-2019-2077

17. Marin, M., Radulescu, V.: A variational approach for the mixed problem in the elastostatics of bodies with dipolar structure. Mediterr. J. Math. 156 (2018)

18. Moameni, A., Salimi, L.: Existence results for supercritical Neumann problem with a convex-concave non-linearity. Ann. Mat. Pura Appl. 198, 1165-1184 (2019)

19. Mokrani, H.: Semilinear sub-elliptic equations on the Heisenberg group with a singular potential. Commun. Pure Appl. Anal. 8, 1619-1636 (2009) 
20. Polya, G., Szego, G.: Problems and Theorems in Analysis, Volum II. Springer, New York (1976)

21. Ragusa, M.A., Tachikawa, A.: Regularity of minimizers of some variational integrals with discontinuity. Z. Anal. Anwend. 27(4), 469-482 (2008)

22. Ragusa, M.A., Tachikawa, A.: Boundary regularity of minimizers of $p(x)$-energy functionals. Ann. Inst. Henri Poincaré, Anal. Non Linéaire 33(2), 451-476 (2016)

23. Razani, A.: Weak and strong detonation profiles for a qualitative model. J. Math. Anal. Appl. 276(2), 868-881 (2002)

24. Razani, A.: Chapman-Jouguet detonation profile for a qualitative model. Bull. Aust. Math. Soc. 66(3), $393-403$ (2002)

25. Razani, A.: Shock waves in gas dynamics. Surv. Math. Appl. 2, 59-89 (2007)

26. Razani, A.: Chapman-Jouguet travelling wave for a two-steps reaction scheme. Ital. J. Pure Appl. Math. 39, 544-553 (2018)

27. Razani, A.: Subsonic detonation waves in porous media. Phys. Scr. 94, 8, 6 pages (2019)

28. Szulkin, A.: Minimax principles for lower semicontinuous functions and applications to nonlinear boundary value problems. Ann. Inst. Henri Poincaré, Anal. Non Linéaire 3(2), 77-109 (1986)

29. Tyagi, J.: Nontrivial solutions for singular semilinear elliptic equations on the Heisenberg group. Adv. Nonlinear Anal. 3, 87-94 (2014)

30. Xiao, Y.-X.: An improved Hardy type inequality on Heisenberg group. J. Inequal. Appl. 2011, 38 (2011) https://doi.org/10.1186/1029-242X-2011-38

\section{Submit your manuscript to a SpringerOpen ${ }^{\circ}$ journal and benefit from:}

- Convenient online submission

- Rigorous peer review

Open access: articles freely available online

High visibility within the field

Retaining the copyright to your article

Submit your next manuscript at $\boldsymbol{~ s p r i n g e r o p e n . c o m ~}$ 